\title{
Impact of the prognostic nutritional index on the recurrence of hepatocellular carcinoma patients after curative resection
}

\author{
Kil Hwan KIM, Sanghyun SONG*, Sungho JO \\ Department of Surgery, Dankook University Hospital, Dankook University College of Medicine, Cheonan, Korea
}

Introduction: The purpose of this retrospective study was to determine the association between the prognostic nutritional index (PNI) and recurrence of hepatocellular carcinoma after curative resection.

Methods: Between 2007 to 2019, 130 patients who underwent curative hepatectomy for hepatocellular carcinoma were enrolled. The PNI was calculated, and the cutoff value was identified through receiver operating characteristic curve analysis. According to the PNI, patients were divided into two groups. Univariate and multivariate analyses were performed to identify independent risk factors for recurrence.

Results: The cutoff value of the PNI was 52. In the univariate analysis, alcoholic liver cirrhosis $(p=0.041)$, protein induced by vitamin $\mathrm{K}$ antagonist-II $\geq 200(p=0.012)$, indocyanine green retention test (ICG R15) $>10 \%(p=0.001)$, estimated blood loss $\geq 800 \mathrm{~mL}(p=$ $0.037)$, tumor size $(p=0.001)$, microvascular invasion $(p=0.023)$, T-stage $(p=0.001)$, and $\mathrm{PNI}<52(p=0.001)$ affected recurrence. In the multivariate analysis, alcoholic liver cirrhosis $(p=0.046)$, ICG R15 $>10 \%(p=0.025)$, T-stage $(p=0.003)$, and PNI $<52(p=0.046)$ were independent prognostic factors for disease-free survival.

Conclusions: The PNI, a nutritional and immunologic factor, is an independent prognostic factor that can predict the recurrence of hepatocellular carcinoma in patients undergoing curative resection. 